\title{
A harmonic/anharmonic energy partition method for lattice statics computations
}

\author{
Rafael Gallego† and Michael Ortiz \\ Division of Engineering, Brown University, Providence, RI 02912, USA \\ Received 8 July 1992, accepted for publication 10 January 1993
}

\begin{abstract}
A method of lattice statics analysis is developed. Consideration of anharmonic effects is restricted to finite regions surrounding lattice defects. All displacements of the crystal are expressed as the effect of unknown forces applied to a perfect harmonic lattice of infinite extent. Displacements are related to the unknown applied forces by means of the Green function of the perfect harmonic lattice, so that equilibrating forces need only be applied to the anharmonic region. The unknown forces are determined so as to maximize the complementary energy of the crystal, which yields a lower bound to the potential energy. The method does not require the explicit enforcement of equilibrium or compatibility conditions across the boundary between the harmonic and anharmonic regions. The performance of the method is assessed on the basis of selected numerical examples. The rate of convergence of the method with increasing domain size is found to be cubic. This is one or two orders of magnitude faster than rigid boundary methods based on the hamonic and continum solutions, respectively.
\end{abstract}

\section{Introduction}

Many mechanical and electronic properties of crystals are strongly influenced by lattice defects such as dislocations. For instance, dislocation cores in microelectronics devices may act either as donor or acceptor-like sites, or behave as high conductance regions (Haansen 1983). In many cases, a detailed knowledge of the atomic structure of the core is required to make quantitative predictions possible. A case in point is provided by the non-planar structure of dislocations in BCC metals, which strongly affects their mobility (Vitek 1992). A similar situation is encountered in the case of dislocations on non-basal planes in $\mathrm{HCP}$ metals, and dislocations in some intermetallic compounds, ordered alloys and non-metallic crystals (see, e.g., Veyssière 1988 for a recent review).

A number of methods for lattice statics computations are presently in existence. At the simplest level of modeling, the lattice may be treated as being harmonic, i.e. as obeying a linear relation between applied forces and displacements (Maradudin 1958, Celli 1961, Boyer and Hardy 1971, Babŭska et al 1960, Heinisch and Sines 1976, Flocken and Hardy 1970, Hölzer and Siems 1970). The resulting system of equilibrium equations is linear and can be conveniently solved by recourse to Fourier analysis or the Green function (Kanzaki 1957, Tewary 1973, Bullough and Tewary 1979). Other methods of analysis allow for anharmonic effects, which strongly influence the structure of the core. In this context, a central computational problem is that of rendering the domain of analysis finite. A frequently used method (see for reviews Vitek 1988, Stoneham et al 1988, Daw 1990) consists of truncating the lattice at some distance from the defect and holding the atoms on the boundary

$\dagger$ Visiting from the Continuum Mechanics Deptartment, ESII, University of Seville, E-41012 Seville, Spain. 
in their isotropic or anisotropic elastic configuration. This, however, can overconstrain the lattice, e.g. by preventing the change of volume attendant to a discrete dislocation. Flexible boundary methods have been proposed which overcome this difficulty, most notably those of Sinclair and co-workers (Sinclair 1971, Sinclair et al 1978, Gehlen et al 1972). However, some features of these approaches contribute to making their implementation onerous. For instance, atoms in the interior and on the boundary of the lattice require very different treatment. In addition, cumbersome equilibrium and compatibility conditions need to be enforced between the lattice and the exterior region.

Here we develop a method of analysis which eliminates some of these shortcomings. In order to effect the requisite reduction to a finite domain, we begin by partitioning the lattice into two regions: one containing the defects, where anharmonic effects are taken into account, or anharmonic region; and the remainder of the crystal, or harmonic region, where the behavior of the lattice is approximated as being harmonic. Because anharmonic effects tend to be confined to small regions surrounding the defects, the numerical error incurred as a result of the harmonic/anharmonic energy partition can be made negligibly small by considering a sufficiently large anharmonic region. It bears emphasis that the anharmonic region need not be simply connected. Indeed, in applications involving multiple defects it may be convenient to surround each defect by disjoint anharmonic subregions. Then the defects interact at a distance through the harmonic lattice.

Secondly, we express all displacements of the crystal as the effect of unknown forces applied to a perfect harmonic lattice of infinite extent. Displacements are related to the unknown applied forces by means of the Green function of the perfect harmonic lattice. In this manner, the equilibrium of the harmonic region is ensured regardless of the choice of forces, which need only be applied to the anharmonic region. The equilibrating forces are determined so as to maximize the complementary energy of the crystal, as computed from the full anharmonic potential. This results in a system of non-linear algebraic equations which we solve by means of Matthies and Strang's (1979) vectorized version of the BFGS method (Dennis and Schnabel 1983, and references therein) preconditioned by the harmonic stiffness matrix. Because the equilibrium equations are derived by rendering the complementary energy of the lattice stationary, the solution bounds the potential energy of the lattice from below. This is in contrast to the more commonly used displacement methods, which result in upper bounds to the potential energy. Both types of method can complement each other in applications where bracketing the energy of defect between tight bounds is of interest.

It should be noted that the method developed here does not require the explicit enforcement of any equilibrium or compatibility conditions across the boundary between the harmonic and anharmonic regions. Indeed, atoms on the boundary of anharmonic region need not be treated any differently from those in the interior. The anharmonic region is merely a truncation device in an otherwise 'seamless' lattice. Tewary (1973) (see also Bullough and Tewary 1979) developed a Green-function method for lattice statics which also possesses this desirable property. However, Tewary's approach is limited in the extent to which anharmonic effects can be taken into account, and does not allow for the consideration of general interaction potentials.

As a numerical test on the method we analyze a Lomer dislocation in silicon using Stillinger and Weber's (1985) potential. This dislocation has been studied in the past (Nandedkar and Narayan 1990), and thus affords direct comparisons with other methods of analysis. We specifically address the issue of convergence in energy as the size of the domain of analysis is allowed to become of infinite extent. Our simulations show that rigid boundary methods based on the anisotropic elasticity solution converge linearly with the number of atoms, while rigid boundary methods based on the harmonic solution exhibit a quadratic 
rate of convergence. By contrast, the method developed here converges at a cubic rate, with the result that the problem size requirements of our method are significantly smaller that those of rigid boundary methods for solutions of comparable accuracy, especially in three dimensions.

\section{Energy considerations}

Throughout this paper we concern ourselves with the stable equilibrium configurations of an atomic lattice. Such configurations constitute local minima of the lattice energy and, therefore, can be determined by energy minimization. We shall assume that a classical potential-energy function $\Phi$ can be defined describing interactions among the $N$ identical atoms of the lattice, and that $\Phi$ admits an expansion into multi-body contributions of the type

$$
\begin{aligned}
\Phi(1, \ldots, N)= & \sum_{l} v_{1}(l)+\sum_{l<l^{\prime}} v_{2}\left(l, l^{\prime}\right)+\sum_{l<l^{\prime}<l^{\prime \prime}} v_{3}\left(l, l^{\prime \prime}, l^{\prime \prime}\right) \\
& +\cdots+v_{N}(1, \ldots, N) .
\end{aligned}
$$

Here, the indices $l, l^{\prime \prime}, l^{\prime \prime}, \ldots$ label the atoms in the lattice and $v_{l}(l), v_{2}\left(l, l^{\prime}\right), v_{3}\left(l, l^{\prime \prime}, l^{\prime \prime}\right)$, $\ldots, v_{N}(1, \ldots, N)$ represent the single-body, two-body, three-body, $\ldots, N$-body terms in the energy expansion. The term $v_{1}(l)$ vanishes in the absence of external fields. The expansion (1) is usually truncated after a few terms. In the special case of semiconductor materials of interest here, the atoms form strong covalent bonds and, consequently, three-body or higher-order interactions must be kept in (1) so that both bond-stretching and bond-bending effects are taken into account. For silicon, an empirical potential including up to three-body interactions was proposed by Stillinger and Weber (1985). The potential has the additive form

$$
\begin{aligned}
& v_{2}\left(r^{l l^{\prime}}\right)=\varepsilon f_{2}\left(r^{l l^{\prime}} / \sigma\right) \\
& v_{3}\left(\boldsymbol{r}^{l}, \boldsymbol{r}^{l^{\prime}}, \boldsymbol{r}^{l^{\prime \prime}}\right)=\varepsilon f_{3}\left(\boldsymbol{r}^{l} / \sigma, \boldsymbol{r}^{l^{\prime}} / \sigma, \boldsymbol{r}^{l^{\prime \prime}} / \sigma\right)
\end{aligned}
$$

where $\epsilon$ and $\sigma$ are energy and length parameters, $r^{l l^{\prime}}$ is the distance between atoms $l$ and $l^{\prime}$, and $\boldsymbol{r}^{\prime}$, denotes the position vector of atoms $l$. The pair potential $f_{2}(r)$ is given by

$$
f_{2}(r)= \begin{cases}A\left(B r^{-p}-r^{-q}\right) \exp \left[(r-a)^{-1}\right] & r<a \\ 0 & r \geqslant a .\end{cases}
$$

The three-body potential has the form

$$
\begin{aligned}
& f_{3}\left(r^{l}, \boldsymbol{r}^{l^{\prime}}, r^{l^{\prime \prime}}\right)=h\left(r^{I^{\prime}}, r^{I^{\prime \prime}}, \theta^{l^{\prime \prime l^{\prime \prime}}}\right)+h\left(r^{l^{\prime \prime}}, r^{l^{\prime \prime}}, \theta^{l^{\prime} l^{\prime \prime}}\right)+h\left(r^{l^{\prime \prime} l}, r^{l^{\prime \prime} l^{\prime}}, \theta^{l^{\prime \prime} l^{\prime}}\right) \\
& h\left(r, r^{\prime}, \theta\right)= \begin{cases}\lambda \exp \left[\gamma(r-a)^{-1}+\gamma\left(r^{\prime}-a\right)^{-1}\right]\left(\cos \theta+\frac{1}{3}\right)^{2} & r, r^{\prime}<a \\
0 & \text { otherwise }\end{cases}
\end{aligned}
$$

where $\theta^{\prime \prime} l^{\prime \prime}$ is the angle between $\boldsymbol{r}^{\prime \prime}$ and $\boldsymbol{r}^{l^{\prime \prime}}$ subtended at node $l$.

Based on careful lattice-dynamics calculations, Stillinger and Weber (1985) optimized the constants of their model to match a wide array of thermomechanical properties of silicon. The resulting values are: $\epsilon=2.1702 \mathrm{eV}, \sigma=2.0951 \AA, A=7.049556277$, 

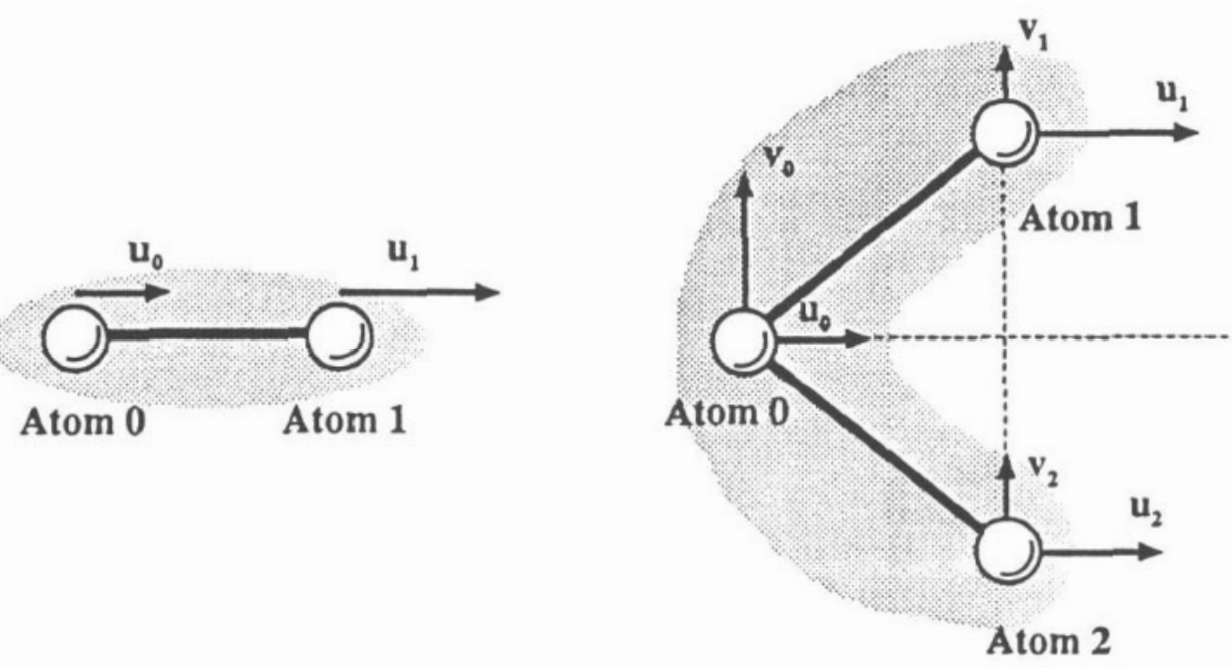

Figure 1. Displacement coordinates for linearization.

$B=0.6022245584, p=4, q=0, a=1.80, \lambda=21.0, \gamma=1.20$. Numerous other potentials for silicon and related materials have been proposed in the literature (Halicioglu et al 1988, Stoneham et al 1988, Duesberry et al 1991). Stillinger and Weber's potential has been successfully employed in a wide range of atomistic simulations (Stillinger and Weber 1985, Landman et al 1986, Abraham and Broughton 1986, Dodson 1986, Nandedkar and Narayan 1990), and will be adopted here. However, it bears emphasis that the numerical procedures developed in this paper apply regardless of the choice of potential.

A situation which frequently is of interest is that of a lattice which is perfect except for isolated defects. Under these conditions, regions of the lattice sufficiently removed from the defects are lightly distorted and their energy can be approximated by a quadratic, or harmonic, potential. The harmonic part of a general potential follows by linearization. Let $\boldsymbol{u}^{l}$ be the displacement of atom $l$ about an equilibrium position $\boldsymbol{r}^{l}$. A Taylor expansion of $\Phi$ in terms of $\boldsymbol{u}^{l}$ gives

$$
\Phi=\Phi_{0}+\Phi_{0}^{\prime}+\Phi_{0}^{\prime \prime}+\cdots
$$

The first term $\Phi_{0}$ is the energy of the lattice at equilibrium. The second term $\Phi_{0}^{\prime}$ vanishes by the equilibrium of the unperturbed configuration. Consequently, to a first approximation, the change in the potential energy is

$$
\Phi_{\mathrm{L}}\left(\boldsymbol{u}^{1}, u^{2}, \ldots, u^{N}\right)=\frac{1}{2} \sum_{l, J^{\prime}=l}^{N} \Phi_{i j}^{l^{\prime}} u_{i}^{l} u_{j}^{l^{\prime}}
$$

where $\Phi_{i j}^{\prime \prime \prime}=\left.\left(\partial^{2} \Phi_{\mathrm{L}} / \partial u_{i}^{l} \partial u_{j}^{l^{\prime}}\right)\right|_{\text {equil. }}$ are stiffness coefficients. Here and subsequently, Einstein's summation convention is adopted on repeated coordinate indices ranging from 1 to 3 . Owing to the ternary terms in the expansion (1), the coefficients $\Phi_{i j}^{l^{\prime}}$ depend on the equilibrium positions of atoms $l$ and $l^{\prime}$ and of all the atoms which have a bond with $l$ and/or $l^{\prime}$, i.e. whose distance to $l$ and/or $l^{\prime}$ in the equilibrium configuration is less than $a$. In the special case of Stillinger and Weber's potential, it is convenient to treat the binary 
and temary terms, (2) and (3) respectively, separately when computing the harmonic part of the potential. With the notation of figure 1 , this gives

$$
\Phi_{\mathrm{II}}=\frac{1}{2} K_{\mathrm{II}}\left(u_{1}-u_{0}\right)^{2}
$$

for two-body interactions, and

$$
\Phi_{\text {III }}=\frac{1}{2} K_{\text {III }}\left(\frac{u_{1}+u_{2}-2 u_{0}}{\sqrt{2}}+\frac{v_{1}-v_{2}}{2}\right)^{2}
$$

for three-body interactions. A simple computation gives $K_{\mathrm{II}}=10.3337 \mathrm{eV} \AA^{-2}$ and $K_{\mathrm{III}}=0.5663 \mathrm{eV} \mathrm{A}^{-2}$ for silicon.
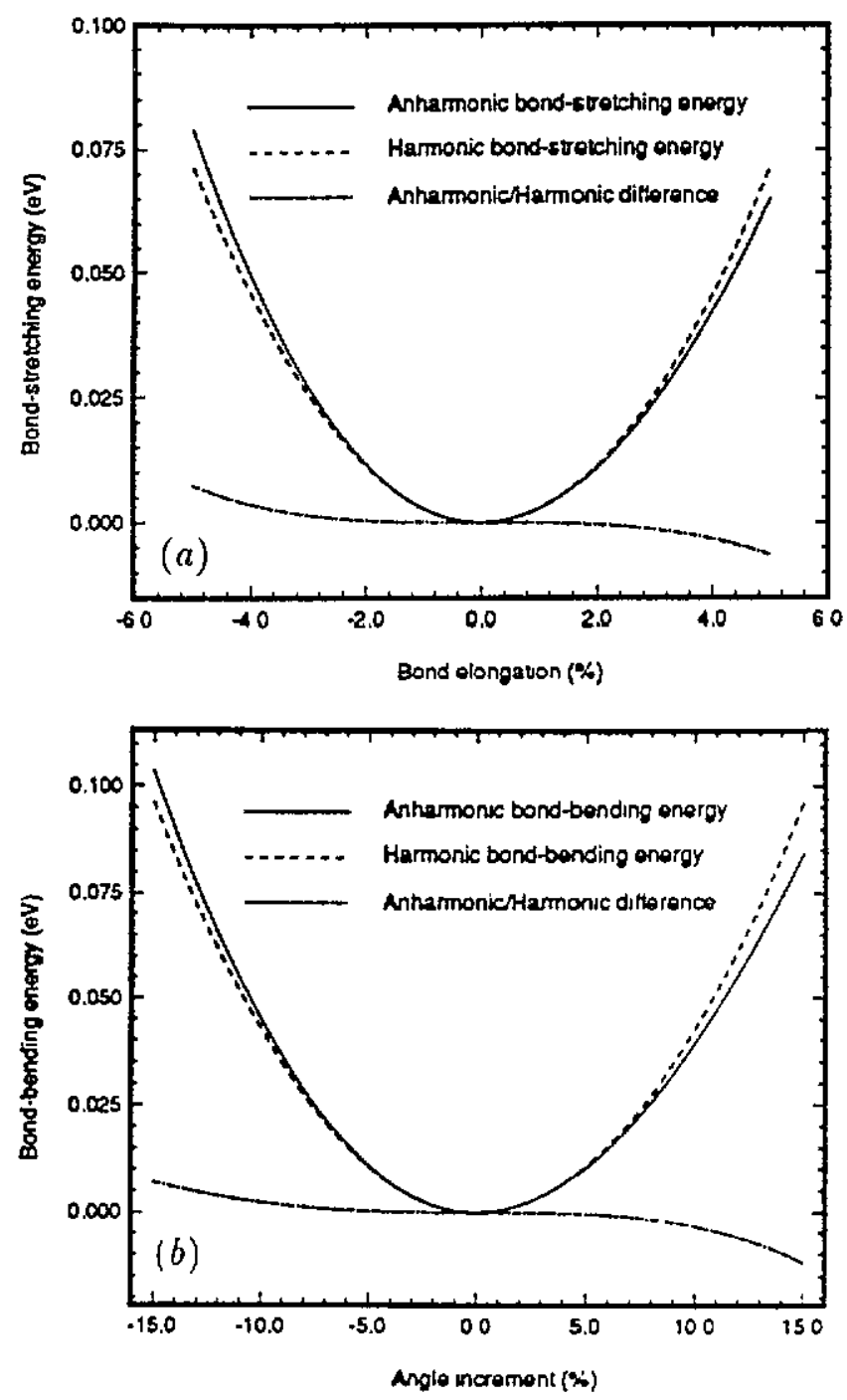

Figure 2. Comparison of anharmonic and harmonic energies. 
Figure 2(a) compares the bond stretching energy (4) in Stillinger and Weber's potential with its harmonic approximation (8). Figure $2(b)$ shows a similar comparison between anharmonic and harmonic non-central three-body interactions due to bond bending, equations (5) and (9), respectively. It is evident from these figures that the anharmonic and harmonic potentials are nearly indistinguishable for small departures from the equilibrium configurations of the bonds.

A calculation of the elastic moduli from the lattice potentials (8) and (9) furnishes a first check on the accuracy of the model. A lengthy but straightforward computation gives

$$
\begin{aligned}
& C_{11}=\frac{K_{\mathrm{II}}+9 K_{\mathrm{III}}}{3 a_{0}} \\
& C_{12}=\frac{2 K_{\mathrm{II}}-9 K_{\mathrm{III}}}{6 a_{0}} \\
& C_{44}=\frac{9 K_{\mathrm{II}} K_{\mathrm{II}}}{2 a_{0}\left(K_{\mathrm{II}}+6 K_{\mathrm{II}}\right)} .
\end{aligned}
$$

Because Stillinger and Weber's potential depends on two constants only, the resulting elastic moduli are related by

$$
\frac{3\left(C_{11}-C_{12}\right)\left(C_{11}+2 C_{12}\right)}{C_{44}\left(7 C_{11}+2 C_{12}\right)}=1 .
$$

For silicon, the actual value of this ratio is equal to 0.85 (Simmons and Wang 1971), 15\% lower than Stillinger and Weber's prediction.

A characteristic property of crystals is that their energy is unaffected by the operation of displacing two half crystals relative to each other in such a way as to leave the lattice invariant. Lack of invariance under this class of transformations constitutes an important deficiency of harmonic potentials. The same problem afflicts continuum treatments of dislocations in solids. In both cases, a convenient device for accounting for the invariance of lattices upon slip is furnished by the concept of eigenstrain (Mura 1977). This concept is central to our subsequent discussion of dislocations in crystals and is developed next.

Making use of the translation invariance of lattice potentials, it is possible to rewrite the harmonic energy of the lattice as (Born and Huang 1954)

$$
\Phi_{\mathrm{L}}=-\frac{1}{2} \sum_{i<l^{t^{\prime}}} \Phi_{i j}^{l l^{\prime}}\left[u_{i}^{l^{\prime}}-u_{i}^{l}\right]\left[u_{j}^{l^{\prime}}-u_{j}^{l^{\prime}}\right]
$$

where the sum extends over all pairs $\left(l, l^{\prime}\right)$ such that $l<l^{\prime}$. Next we write the relative displacements between pairs of atoms as

$$
\begin{aligned}
& u_{i}^{l^{\prime}}-u_{i}^{l}=\left[\beta_{j i}^{l^{\prime}}+\bar{\beta}_{j i}^{l l^{\prime}}\right] r_{j}^{l^{\prime \prime}} \\
& r_{j}^{l^{\prime}}=r_{j}^{l^{\prime}}-r_{j}^{l}
\end{aligned}
$$

where the matrices $\left(\beta_{j i}^{\prime l^{\prime}}+\bar{\beta}_{j i}^{\prime l^{\prime}}\right)$ define local affine transformations which describe the distorsion of the lattice. We shall refer to $\beta_{j i}^{l l^{\prime}}$ as the elastic, or reversible, distorsion of the lattice, and to $\bar{\beta}_{j i}^{l l^{\prime}}$ as the eigendistorsion. The symmetric parts of $\beta_{j i}^{\prime l^{\prime}}$ and $\bar{\beta}_{j i}^{\prime l^{\prime}}$ may 
be thought of as defining the elastic strain and the eigenstrain, respectively, by analogy to continuum mechanics.

The defining property of the eigendistorsion $\bar{\beta}_{j i}^{\prime \prime}$ is that it leaves the energy of the lattice invariant. Consequently, the harmonic lattice energy can be expressed as a function of $\beta_{j i}^{\prime l^{\prime}}$ only, i.e.

$$
\Phi_{\mathrm{L}}=-\frac{1}{2} \sum_{l<l^{\prime}} \Phi_{i j}^{l^{\prime}}\left(\beta_{k i}^{l^{\prime}} r_{k}^{l^{\prime}}\right)\left(\beta_{m j}^{l^{\prime}} r_{m}^{l^{\prime}}\right)
$$

which can be rewritten as

$$
\Phi_{\mathrm{L}}=\frac{1}{2} \sum_{l, l^{\prime}} \Phi_{i j}^{l^{\prime}} u_{i}^{l} u_{j}^{l^{\prime}}-\sum_{l, l^{\prime}} \Phi_{i j}^{l^{\prime}} \bar{\beta}_{k j}^{l^{\prime}} r_{k}^{l^{\prime}} u_{i}^{l} .
$$

The forces which need to be applied to a harmonic lattice to induce a given distribution of eigenstrains can be computed by differentiating (15) with respect to $u_{i}^{l}$, with the result

$$
\begin{aligned}
& F_{L^{\prime}}^{l}=-\frac{\partial \Phi_{\mathrm{L}}}{\partial u_{i}^{l}}=-\sum_{l^{\prime}} \Phi_{i j}^{l^{\prime}} u_{j}^{l^{\prime}}+\mathcal{F}_{i}^{\prime} \\
& \mathcal{F}_{i}^{\prime}=\sum_{l^{\prime}} \Phi_{i j}^{l l^{\prime}} \bar{\beta}_{k j}^{l^{\prime}} r_{k}^{l^{\prime}} .
\end{aligned}
$$

By analogy with the theory of linear elastic defects, (17) may be thought of as defining a distribution of body forces, or 'eigenforces', over the lattice.

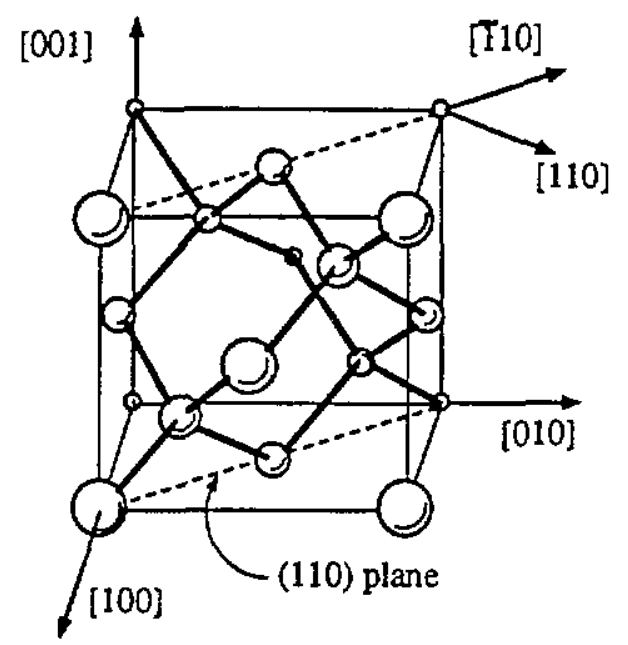

Figure 3. Cubic unit cell for silicon.

\section{Harmonic lattice statics}

We have shown in the foregoing that lattice defects in a harmonic lattice can be simulated by way of suitably defined eigenforces. Because these forces are applied to a perfect harmonic lattice, the corresponding displacement field can be conveniently computed with the aid of 
the Green function. Similarly, in subsequent derivations we shall account for anharmonic effects near the core of defects by applying a suitable distribution of forces to an otherwise harmonic lattice. Here again, the Green function for the harmonic lattice can be conveniently employed to determine the ensuing displacements. In this section we succinctly present a general method for calculating the Green function of a harmonic lattice (Tewary 1973). Of special interest in some applications, such as Lomer dislocations in silicon, is the twodimensional Green function, i.e. the displacement field due to an infinite row of unit point forces. The computation of this Green function presents additional difficulties related to the emergence of logarithmic singularities. The appropriate treatment of these singularities is also discussed in some detail.

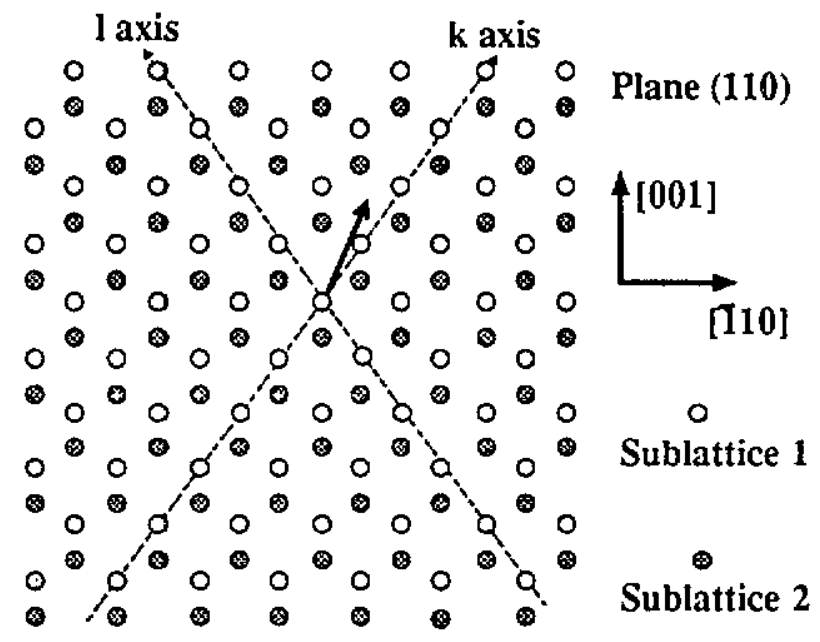

Figure 4. View of plane (110) in silicon.

For definiteness, we confine our discussion to lattices possessing the diamond structure, figure 3 , and to Stillinger and Weber's potential, the harmonic part of which is given by (8) and (9). With reference to figure 4, the in-plane displacements in static equilibrium with an infinite row of point forces satisfy the system of equations:

$$
\begin{aligned}
& f_{k l}^{1}=\frac{K_{\mathrm{II}}}{3}\left(4 u_{k l}^{1}\right.\left.-2 u_{(k+1) l}^{2}-2 u_{k(l+1)}^{2}-\sqrt{2} v_{(k+1) l}^{2}+\sqrt{2} v_{k(l+1)}^{2}\right) \\
&+\frac{K_{\mathrm{II}}}{8}\left(56 u_{k l}^{1}+3 u_{(k-1) l}^{1}+3 u_{k(l+1)}^{1}-2 u_{(k-1)(l+1)}^{1}-8 u_{(k+1) l}^{2}\right. \\
&-48 u_{k l}^{2}-8 u_{k(l+1)}^{2}-2 u_{(k+1)(l-1)}^{1}+3 u_{(k+1) l}^{1}+3 u_{k(l-1)}^{1} \\
&-3 \sqrt{2} v_{(k-1) l}^{1}-\sqrt{2} v_{k(l+1)}^{1}+2 \sqrt{2} v_{(k-1)(l+1)}^{1}+8 \sqrt{2} v_{(k+1) l}^{2} \\
&\left.-8 \sqrt{2} v_{k(l+1)}^{2}-2 \sqrt{2} v_{(k+1)(l-1)}^{1}+\sqrt{2} v_{(k+1) l}^{\mathrm{I}}+3 \sqrt{2} v_{k(l-1)}^{1}\right) \\
& g_{k l}^{1}=-\frac{K_{\Pi}}{3}\left(\sqrt{2} u_{(k+1) l}^{2}-\sqrt{2} u_{k(l+1)}^{2}-4 v_{k l}^{1}+v_{(k+1)}^{2}+2 v_{k l}^{2}+v_{k(l+1)}^{2}\right) \\
&+\frac{K_{\mathrm{III}}}{8}\left(\sqrt{2} u_{(k-1) l}^{1}+3 \sqrt{2} u_{k(l+1)}^{1}-2 \sqrt{2} u_{(k-1)(l+1)}^{1}+8 \sqrt{2} u_{(k+1) l}^{2}\right. \\
&-8 \sqrt{2} u_{k(l+1)}^{2}+2 \sqrt{2} u_{(k+1)(l-1)}^{1}-3 \sqrt{2} u_{(k+1) l}^{1}-\sqrt{2} u_{k(l-1)}^{1} \\
&+64 v_{k l}^{1}-2 v_{(k-1) l}^{1}-2 v_{k(l+1)}^{1}+4 v_{(k-1)(l+1)}^{1}-16 v_{(k+1) l}^{2} \\
&\left.-32 v_{k l}^{2}-16 v_{k(l+1)}^{2}+4 v_{(k+1)(l-1)}^{1}-2 v_{(k+1) l}^{1}-2 v_{k(l-1)}^{1}\right)
\end{aligned}
$$


where $u_{k l}^{i}$ and $v_{k l}^{i}$ are the Cartesian components of the displacement vector at a site of skew lattice coordinates $(m, n)$, and the the index $i=1,2$ labels each of the two face centered cubic sublattices which make up the diamond structure. The terms $f_{k l}^{l}$ and $g_{k l}^{1}$ are cartesian components of the external forces applied at point $(k, l)^{\mathrm{I}}$. Two similar equations apply when the point loads act on the second sublattice. Equations (18) and (19) follow simply by rendering the harmonic energy of the lattice stationary.

Two-dimensional Fourier series furnish a convenient and powerful device for solving equations (18) and (19). From the discrete displacements $u_{k l}^{i}$ and $v_{k l}^{i}(i=1,2)$, a pair of complex functions of two complex variables can be defined as

$$
\begin{aligned}
& U^{\prime}\left(\xi^{\prime}, \eta^{\prime}\right)=\sum_{k=-\infty}^{\infty} \sum_{l=-\infty}^{\infty} u_{k l}^{j} \mathrm{e}^{-\mathrm{i}\left(k \xi^{\prime}+l \eta^{\prime}\right)} \\
& V^{j}\left(\xi^{\prime}, \eta^{\prime}\right)=\sum_{k=-\infty}^{\infty} \sum_{l=-\infty}^{\infty} v_{k l}^{j} \mathrm{e}^{-\mathrm{i}\left(k \xi^{\prime}+l \eta^{\prime}\right)} .
\end{aligned}
$$

Likewise, from the discrete applied forces we define

$$
\begin{aligned}
& F^{j}\left(\xi^{\prime}, \eta^{\prime}\right)=\sum_{k=-\infty}^{\infty} \sum_{l=-\infty}^{\infty} f_{k l}^{j} \mathrm{e}^{-\mathrm{i}\left(k \xi^{\prime}+l \eta^{\prime}\right)} \\
& G^{j}\left(\xi^{\prime}, \eta^{\prime}\right)=\sum_{k=-\infty}^{\infty} \sum_{l=-\infty}^{\infty} g_{k l}^{j} \mathrm{e}^{-\mathrm{i}\left(k \xi^{\prime}+l \eta^{\prime}\right)} .
\end{aligned}
$$

The original displacement and force fields are recovered by multiplying both sides of (20) and $(21)$ by $\mathrm{e}^{\mathrm{i}\left(k^{\prime} \xi^{\prime}+l^{\prime} \eta^{\prime}\right)}$ and integrating over the square domain $\left(\xi^{\prime}, \eta^{\prime}\right) \in[-\pi, \pi] \times[-\pi, \pi]$. Recalling that

$$
\int_{-\pi}^{\pi} \int_{-\pi}^{\pi} \mathrm{e}^{-\mathrm{i}\left(m \xi^{\prime}+n \eta^{\prime}\right)}=4 \pi^{2} \delta_{m 0} \delta_{n 0}
$$

the inverse transforms follow as

$$
\begin{aligned}
& u_{k l}^{j}=\frac{1}{4 \pi^{2}} \int_{-\pi}^{\pi} \int_{-\pi}^{\pi} U^{j}\left(\xi^{\prime}, \eta^{\prime}\right) \mathrm{e}^{\mathrm{i}\left(k \xi^{\prime}+l \eta^{\prime}\right)} \mathrm{d} \xi^{\prime} \mathrm{d} \eta^{\prime} \\
& v_{k l}^{j}=\frac{1}{4 \pi^{2}} \int_{-\pi}^{\pi} \int_{-\pi}^{\pi} V^{j}\left(\xi^{\prime}, \eta^{\prime}\right) \mathrm{e}^{\mathrm{i}\left(k \xi^{\prime}+l \eta^{\prime}\right)} \mathrm{d} \xi^{\prime} \mathrm{d} \eta^{\prime}
\end{aligned}
$$

and

$$
\begin{aligned}
& f_{k l}^{j}=\frac{1}{4 \pi^{2}} \int_{-\pi}^{\pi} \int_{-\pi}^{\pi} F^{j}\left(\xi^{\prime}, \eta^{\prime}\right) \mathrm{e}^{\mathrm{i}\left(k \xi^{\prime}+l \eta^{\prime}\right)} \mathrm{d} \xi^{\prime} \mathrm{d} \eta^{\prime} \\
& g_{k l}^{j}=\frac{1}{4 \pi^{2}} \int_{-\pi}^{\pi} \int_{-\pi}^{\pi} G^{j}\left(\xi^{\prime}, \eta^{\prime}\right) \mathrm{e}^{\mathrm{j}\left(k \xi^{\prime}+l \eta^{\prime}\right)} \mathrm{d} \xi^{\prime} \mathrm{d} \eta^{\prime}
\end{aligned}
$$

The applied forces of interest here vanish everywhere except at $(0,0)^{1}$ and $(0,0)^{2}$. The corresponding Fourier transforms are, therefore,

$$
\begin{aligned}
& F^{j}\left(\xi^{\prime}, \eta^{\prime}\right)=f^{j} \\
& G^{j}\left(\xi^{\prime}, \eta^{\prime}\right)=g^{j}
\end{aligned}
$$


where $\left(f^{j}, g^{j}\right)$ are the forces applied at $(0,0)^{j}$. Inserting (23) and (24) into the equilibrium equations (18), (19), as well as into the analogous equations for the second sublattice, the system of algebraic equations

$$
\left(\begin{array}{llll}
m_{11} & m_{12} & m_{13} & m_{14} \\
\bar{m}_{12} & m_{11} & \bar{m}_{14} & \bar{m}_{13} \\
\bar{m}_{13} & m_{14} & m_{33} & m_{34} \\
\bar{m}_{14} & m_{13} & \bar{m}_{34} & m_{33}
\end{array}\right)\left(\begin{array}{c}
U^{1}\left(\xi^{\prime}, \eta^{\prime}\right) \\
U^{2}\left(\xi^{\prime}, \eta^{\prime}\right) \\
V^{1}\left(\xi^{\prime}, \eta^{\prime}\right) \\
V^{2}\left(\xi^{\prime}, \eta^{\prime}\right)
\end{array}\right)=\left(\begin{array}{l}
f^{1} \\
f^{2} \\
g^{1} \\
g^{2}
\end{array}\right)
$$

in the variables $U^{j}\left(\xi^{\prime}, \eta^{\prime}\right)$ and $V^{j}\left(\xi^{\prime}, \eta^{\prime}\right)$ is obtained. Here, the notation $\bar{m}_{i j}$ means the complex conjugate of $m_{i j}$. The matrix coefficients $m_{i j}$ in (26) are

$$
\begin{aligned}
& m_{11}=\frac{4}{3} K_{\mathrm{II}}+\frac{1}{8} K_{\mathrm{III}}\left(60+3 e_{1}+3 e_{2}-\frac{e_{1}+e_{2}}{e_{1} e_{2}}\left(e_{1}+e_{2}-3\right)\right) \\
& m_{12}=-\frac{2}{3} K_{\mathrm{II}}\left(e_{1}+e_{2}\right)-K_{\mathrm{II}}\left(e_{1}+e_{2}-6\right) \\
& m_{13}=\frac{\sqrt{2}}{8} K_{\mathrm{II}} \frac{-e_{1}+e_{2}}{e_{1} e_{2}}\left(2 e_{1}+2 e_{2}-e_{1} e_{2}-3\right) \\
& m_{14}=-\frac{\sqrt{2}}{3}\left(K_{\mathrm{II}}-3 K_{\mathrm{II}}\right)\left(e_{1}-e_{2}\right) \\
& m_{33}=\frac{4}{3} K_{\mathrm{II}}+\frac{1}{4} K_{\mathrm{III}}\left(28-e_{1}-e_{2}+\frac{e_{1}+e_{2}}{e_{1} e_{2}}\left(2 e_{1}+2 e_{2}-3\right)\right) \\
& m_{34}=-\frac{1}{3}\left(K_{\mathrm{II}}+6 K_{\mathrm{III}}\right)\left(2+e_{1}+e_{2}\right) \\
& e_{1}=\exp \left(\mathrm{it}^{\prime}\right) \\
& e_{2}=\exp \left(\mathrm{in}^{\prime}\right) .
\end{aligned}
$$

With the help of a symbolic processor program (Wolfram 1991), the above system is readily solved, with the result

$$
\left(\begin{array}{l}
U^{1}\left(\xi^{\prime}, \eta^{\prime}\right) \\
U^{2}\left(\xi^{\prime}, \eta^{\prime}\right) \\
V^{\prime}\left(\xi^{\prime}, \eta^{\prime}\right) \\
V^{2}\left(\xi^{\prime}, \eta^{\prime}\right)
\end{array}\right)=\frac{1}{D\left(\xi^{\prime}, \eta^{\prime}\right)}\left(\begin{array}{llll}
r_{11} & r_{12} & r_{13} & r_{14} \\
\bar{r}_{12} & r_{14} & \bar{r}_{14} & \bar{r}_{13} \\
\bar{r}_{13} & r_{14} & r_{33} & r_{34} \\
\bar{r}_{14} & r_{13} & \bar{r}_{34} & r_{33}
\end{array}\right)\left(\begin{array}{l}
f^{1} \\
f^{2} \\
g^{1} \\
g^{2}
\end{array}\right)
$$

where $r_{i j}\left(\xi^{\prime}, \eta^{\prime}\right)$ and $D\left(\xi^{\prime}, \eta^{\prime}\right)$ are lengthy polynomials in the variables $\cos \xi^{\prime}, \sin \xi^{\prime}, \cos \eta^{\prime}$ and $\sin \eta^{\prime}$. The function $D\left(\xi^{\prime}, \eta^{\prime}\right)$ is the determinant of the matrix $\left(m_{i j}\right)$, so that the identity $m_{i j}^{-1}=r_{i j} / D$ holds.

The sought displacement field follows by effecting the inverse Fourier transform (23). In so doing, a first difficulty that is encountered is that the resulting integrals diverge. However, the integrals can be rendered convergent simply by adding a rigid translation to the displacement field. Evidently, the solution so obtained is also in equilibrium. A suitable choice of rigid translation is

$$
\left(\begin{array}{l}
U_{0}^{1}\left(\xi^{\prime}, \eta^{\prime}\right) \\
U_{0}^{2}\left(\xi^{\prime}, \eta^{\prime}\right) \\
V_{0}^{1}\left(\xi^{\prime}, \eta^{\prime}\right) \\
V_{0}^{2}\left(\xi^{\prime}, \eta^{\prime}\right)
\end{array}\right)=-\frac{1}{D} \mathrm{e}^{-\mathrm{i}\left(k \xi^{\prime}+3 \eta^{\prime}\right)}\left(\begin{array}{cccc}
r_{11} & r_{11} & 0 & 0 \\
r_{11} & r_{11} & 0 & 0 \\
0 & 0 & r_{33} & r_{33} \\
0 & 0 & r_{33} & r_{33}
\end{array}\right)\left(\begin{array}{l}
f^{l} \\
f^{2} \\
g^{1} \\
g^{2}
\end{array}\right)
$$


The resulting integral expressions, while convergent, are still difficult to evaluate owing to the rapidly oscillatory character of the integrands, especially when displacements are computed at sites which are distant from the point of application of the loads. Because of the symmetry of the integrands with respect to the axes $\xi^{\prime}=\eta^{\prime}$ and $\xi^{\prime}=-\eta^{\prime}$ all integrals can be reduced to the triangle $\left\{\xi^{\prime}-\eta^{\prime} \geqslant 0 ; \xi^{\prime}+\eta^{\prime} \geqslant 0 ; \eta^{\prime} \leqslant \pi\right\}$. We have found that a product Grauss quadrature rule (Stroud, 1971) on $80 \times 80$ points yields displacements accurate to within $10^{-6}$ in the region $k, l \leqslant 40$.
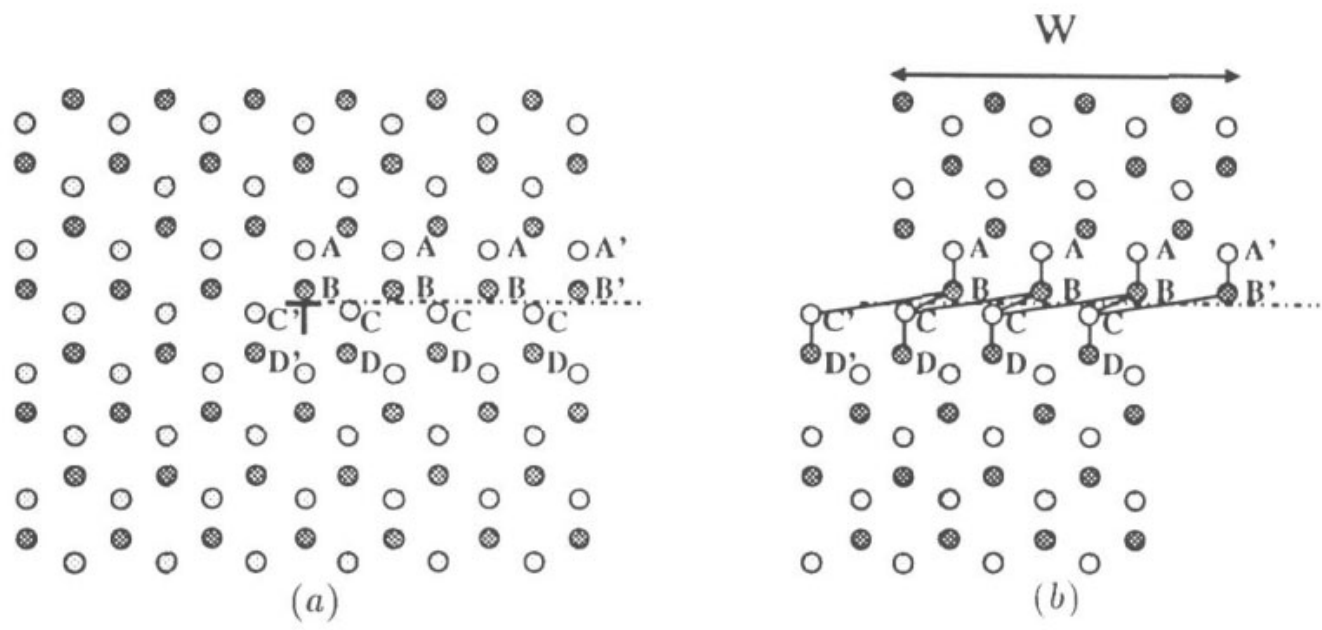

Figure 5. Calculation of eigenforces: (a) Cutting plane in the perfect lattice, (b) equivalent eigendistorsions.

Calculations of the displacement fields of dislocations in harmonic lattices provide an example of application of the methodology described in previous sections. Consideration of anharmonic effects is deferred to section 4 . We begin by computing the eigenforces $\mathcal{F}_{i}^{l}$ (17) corresponding to a Lomer edge dislocation in silicon. This particular dislocation may be thought of as the result of cutting the crystal through a half plane of the (001) type, the edge of the cut, or dislocation line, lying on the [110] direction, and subsequently displacing the upper half of the crystal by a distance $b=(\sqrt{2} / 2) a_{0}$ in the direction [110]. With reference to figure 5 , the corresponding eigenforces are computed to be:

$\begin{array}{llll}\mathcal{F}_{x}^{\mathrm{A}}=-F_{x}^{2} & \mathcal{F}_{y}^{\mathrm{A}}=0.0 & \mathcal{F}_{x}^{\mathrm{B}}=F_{x}^{1} & \mathcal{F}_{y}^{\mathrm{B}}=0.0 \\ \mathcal{F}_{x}^{\mathrm{C}}=-F_{x}^{1} & \mathcal{F}_{y}^{\mathrm{C}}=0.0 & \mathcal{F}_{x}^{\mathrm{D}}=F_{x}^{2} & \mathcal{F}_{y}^{\mathrm{D}}=0.0 \\ \mathcal{F}_{x}^{\mathrm{A}^{\prime}}=-F_{x}^{2} / 2 & \mathcal{F}_{y}^{\mathrm{A}^{\prime}}=-F_{y}^{2} & \mathcal{F}_{x}^{\mathrm{B}^{\prime}}=F_{x}^{1} / 2 & \mathcal{F}_{y}^{\mathrm{B}^{\prime}}=F_{y}^{1} \\ \mathcal{F}_{x}^{\mathrm{C}^{\prime}}=-F_{x}^{1} / 2 & \mathcal{F}_{y}^{\mathrm{C}^{\prime}}=-F_{y}^{1} & \mathcal{F}_{x}^{\mathrm{D}^{\prime}}=F_{x}^{2} / 2 & \mathcal{F}_{y}^{\mathrm{D}^{\prime}}=F_{y}^{2}\end{array}$

where $F_{x}^{1}=55.57 \mathrm{eV} \AA^{-1}, F_{x}^{2}=1.63 \mathrm{eV} \AA^{-1}, F_{y}^{1}=18.30 \mathrm{eV} \AA^{-1}, F_{y}^{2}=0.38 \mathrm{eV} \AA^{-1}$. This system of forces is self-equilibrated. Isolated dislocations follow as the limit of $W \rightarrow \infty$, figure 5 .

The displacement field due to this system of eigenforces can be computed by superposition of the point-load solutions derived in the preceding section. For convenience, we group the eigenforces into multipoles of zero resultant force and moment. The structure of these multipoles is shown in figure 6. The forces at $\mathrm{C}^{\prime \prime}$ and $\mathrm{D}^{\prime \prime}$ are $\mathcal{F}_{x}^{\mathrm{C}^{\prime \prime}}=-F_{x}^{1} / 2, \mathcal{F}_{y}^{\mathrm{C}^{\prime \prime}}=$ 


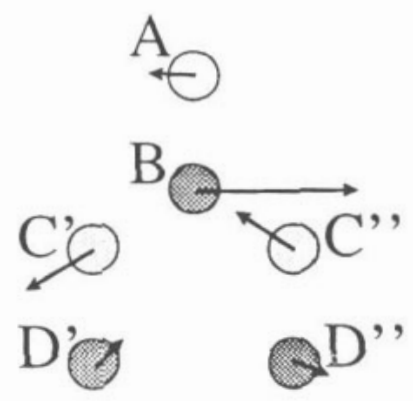

Figure 6. Structure of multipoles for dislocation calculation.

$F_{y}^{1}, \mathcal{F}_{x}^{\mathrm{D}^{\prime \prime}}=F_{x}^{2} / 2, \mathcal{F}_{y}^{\mathrm{D}^{\prime \prime}}=-F_{y}^{2}$, respectively. The complete system of eigenforces consists of a semi-infinite row of multipoles with points $\mathrm{C}^{\prime}$ at locations $(k,-k)^{1}, k=0 \ldots, \infty$.

The displacements due to two multipoles centered at $\left(s^{\prime},-s^{\prime}\right)^{1}$ are

$$
u_{k l s^{\prime}}^{j}=\sum_{i=1}^{3} \frac{1}{4 \pi^{2}} \int_{-\pi}^{\pi} \int_{-\pi}^{\pi} \frac{r_{j h}\left(\xi^{\prime}, \eta^{\prime}\right)}{D\left(\xi^{\prime}, \eta^{\prime}\right)} f_{i}^{h} \mathrm{e}^{\left.\mathrm{i}\left(k-k_{r}^{\prime}\right) \xi^{\prime}+\left(l-l_{r}^{\prime}\right) \eta^{\prime}\right)} \mathrm{d} \xi^{\prime} \mathrm{d} \eta^{\prime}
$$

where, for simplicity of notation, the indices $j$ and $h$ now range from 1 to 4 , the index $t$ pertains to the three pairs of atoms where the forces are applied, and $\left(k_{t}^{\prime}, l_{t}^{\prime}\right)$ are the lattice coordinates of those pairs. At this point it proves convenient to introduce new variables of integration $\xi=\frac{1}{2}\left(\xi^{\prime}+\eta^{\prime}\right)$ and $\eta=\frac{1}{2}\left(\xi^{\prime}-\eta^{\prime}\right)$ whereupon (31) becomes

$$
u_{k l s}^{j}=\sum_{t=1}^{3} \frac{1}{2 \pi^{2}} \int_{\mathcal{A}} \int \frac{r_{j h}(\xi, \eta)}{D(\xi, \eta)} f_{t}^{h} \mathrm{e}^{\mathrm{i} i\left(m-m_{s}^{\prime}\right) \xi+\left(n-n_{t}^{\prime}\right) \eta \eta} \mathrm{d} \xi \mathrm{d} \eta .
$$

New and old lattice indices are related by $m=k+l$ and $n=k-l$. The new indices give the discrete cartesian coordinates of the atoms directly. The coordinates of points $\mathrm{C}$ in the distribution of eigenforces, figure 6 , are $\left[m_{C}, n_{C}\right]=\left[0,2 s^{\prime}\right]=[0, s]$; the relative coordinates of the remaining points of application of forces are $\left[m_{t}^{\prime}, n_{t}^{\prime}\right]=\left[m_{t}, s+n_{t}\right]$, where $m_{\mathrm{A}^{\prime}}=m_{\mathrm{B}^{\prime}}=m_{\mathrm{A}^{\prime \prime}}=m_{\mathrm{B}^{\prime \prime}}=1, n_{\mathrm{A}^{\prime}}=n_{\mathrm{B}^{\prime}}=-1, m_{A^{\prime \prime}}=m_{\mathrm{B}^{\prime \prime}}=1$ and $m_{\mathrm{C}}=m_{\mathrm{D}}=n_{\mathrm{C}}=n_{\mathrm{D}}=0$. The area of integration $\mathcal{A}$ is the square in the plane $(\xi, \eta)$ of vertices $(\pi, 0),(0, \pi),(-\pi, 0)$ and $(0,-\pi)$.

Interchanging the order of the sums and integrals, equation (31) becomes

$$
\begin{gathered}
u_{k l s}^{j}=\frac{1}{2 \pi^{2}} \int_{A} \int \frac{r_{j h}(\xi, \eta)}{D(\xi, \eta)} \sum_{t=1}^{3}\left\{f_{h t} \mathrm{e}^{-\mathrm{i}(m, \xi+n, \eta)}\right\} \mathrm{e}^{\mathrm{i}(m \xi+n \eta)} \mathrm{e}^{-\mathrm{i} s \eta]} \mathrm{d} \xi \mathrm{d} \eta \\
=\frac{1}{2 \pi^{2}} \int_{\mathcal{A}} \int \frac{r_{j h}(\xi, \eta)}{D(\xi, \eta)} F_{h}(\xi, \eta) \mathrm{e}^{\mathrm{i}(m \xi+n \eta)} \mathrm{e}^{-\mathrm{i} s \eta} \mathrm{d} \xi \mathrm{d} \eta
\end{gathered}
$$

where

$$
F_{h}(\xi, n)=\sum_{i=1}^{7}\left\{f_{h t} \mathrm{e}^{-\mathrm{i}\left(m_{t} \xi+n, \eta\right)}\right\}
$$


The complete displacement field $u_{k l}^{i}$ of the dislocation now follows by superposition of multipole displacements $u_{k l s}^{i}$, which yields

$$
u_{k l}^{j}=\sum_{s=0}^{\infty} u_{k l s}^{j}
$$

where $\Sigma^{\prime}$ denotes summation over all even integers $s$. Substituting (33) into (35) gives

$$
\begin{aligned}
u_{k l}^{j}=\sum_{s=0}^{\infty} \frac{1}{2 \pi^{2}} \int_{\mathcal{A}} \int \frac{r_{j h}(\xi, \eta)}{D(\xi, \eta)} F_{h}(\xi, \eta) \mathrm{e}^{\mathrm{i}(m \xi+n \eta)} \mathrm{e}^{-\mathrm{i} s \eta} \mathrm{d} \xi \mathrm{d} \eta \\
=\frac{1}{2 \pi^{2}} \int_{\mathcal{A}} \int \frac{r_{j h}(\xi, \eta)}{D(\xi, \eta)} F_{h}(\xi, \eta) \mathrm{e}^{\mathrm{i}(m \xi+n \eta)} \sum_{s=0}^{\infty} \mathrm{e}^{-\mathrm{i} s \eta} \mathrm{d} \xi \mathrm{d} \eta .
\end{aligned}
$$

The sum in the last integral can be summed explicitly (Jones 1967), with the result

$$
\sum_{s=0}^{\infty} \mathrm{e}^{-\mathrm{i} s \eta}=\frac{\pi}{2} \sum_{m=-\infty}^{\infty} \delta(\eta+m \pi)-\frac{\mathrm{ie}^{\mathrm{i} \eta}}{2 \sin \eta}
$$

where $\delta(\eta)$ is Dirac's delta function. By the properties of the delta function, (36) transforms into

$$
\begin{aligned}
u_{k l s}^{j}=\frac{1}{4 \pi} \int_{-\pi}^{\pi} & \frac{r_{j h}(\xi, 0)}{D(\xi, 0)} F_{h}(\xi, 0) \mathrm{e}^{\mathrm{i} m \xi} d \xi \\
& -\frac{\mathrm{i}}{4 \pi^{2}} \int_{\mathcal{A}} \int \frac{r_{j h}(\xi, \eta)}{D(\xi, \eta) \sin \eta} F_{h}(\xi, \eta) \mathrm{e}^{[! m \xi+(n+1) \eta]} \mathrm{d} \xi \mathrm{d} \eta .
\end{aligned}
$$

Both integrands have singularities of order $r^{-1}$, so that the corresponding integrals are defined in the Cauchy principal value sense. The integrand in the first integral of (38) has an isolated singularity at $\xi=0$, while the remaining integrand has a line of singularities along $\eta=0$. Because of the antisymmetry of the imaginary part of the integrand, the imaginary part of the first integral in (38) vanishes identically. Similarly, the real part of the second integral is zero. The remaining integrals are convergent and can be computed readily by Gaussian quadrature. By taking advantage of the double symmetry of the integrand with respect to both $\xi$ and $\eta$, the domain of the double integral can be reduced to one quarter of the original square domain.

Equation (38) provides an analytical representation of the equilibrium configuration of a Lomer edge dislocation in silicon, as computed within the harmonic approximation. Figure 7 shows the structure of the core. Figure 8 compares the lattice statics and the anisotropic elasticity solutions (Teodosiu 1982). A few atomic distances away from the core, the agreement between both displacement fields is remarkable. It should be carefully noted, however, that the covergence of the 'strain' field, as measured by differences in nextneighbor displacements, is far slower. This circumstance greatly diminishes the accuracy of fixed boundary methods based on the continuum solution, as demonstrated in section 5 .

It bears emphasis that the lack of symmetry in the harmonic and continuum solutions depicted in figures 7 and 8 is unphysical, and stems from the breakdown of linear approximations near the core. Thus, in the harmonic approximation, equilibrium is established with reference to the undeformed lattice, and, because the eigenstrains used to introduce the dislocation are unsymmetrical, so is the resulting harmonic solution. This situation resolves itself when non-linear effects are fully taken into account, as shown in section 4. By enforcing equilibrium in the deformed configuration of the lattice and taking the full anharmonic potential into account, the core solution then exhibits the full symmetry of the parent lattice. 


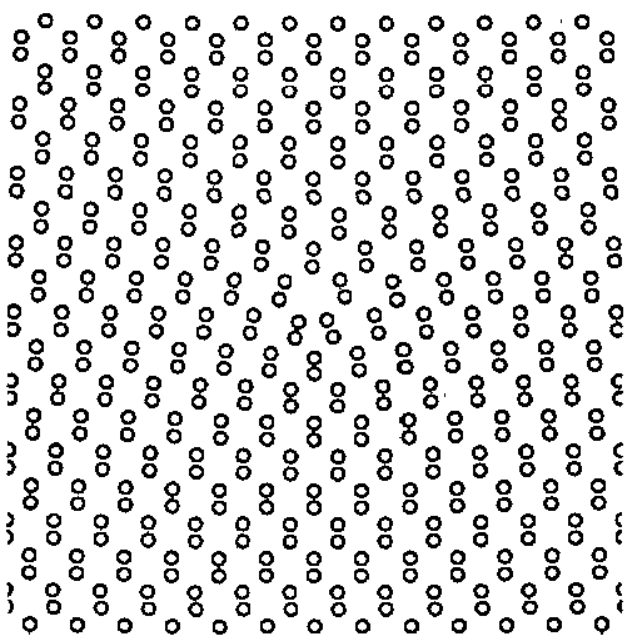

Figure 7. Lomer dislocation in silicon: harmonic approximation.

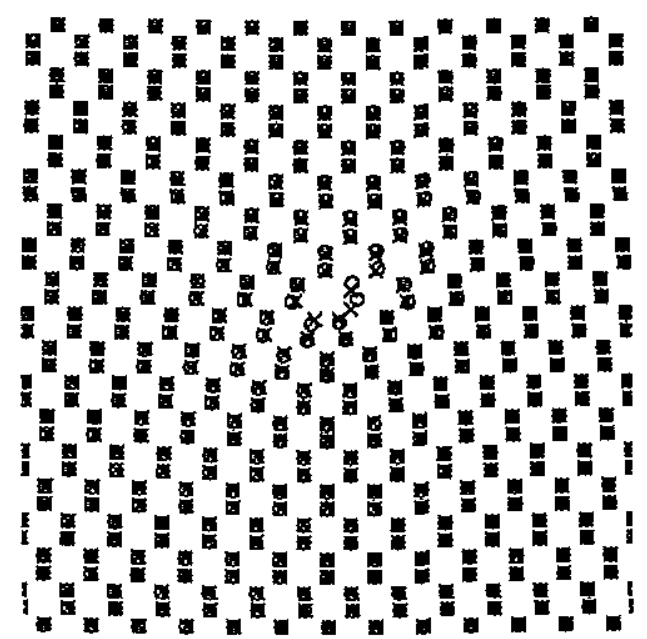

Figure 8. Lomer dislocation in silicon: comparison of lattice $(0)$ and continuum $(x)$ solutions.

\section{Anharmonic lattice statics}

Near the core of lattice defects the full interaction potential, as opposed to merely its harmonic part, may be expected to come into play, and anharmonic effects need to be taken into account. Frequently, however, anharmonic effects are confined to a rather small region surrounding the defect. Outside this region, the anharmonicity of the potential can be altogether neglected, and the harmonic formulation developed in the preceding section applies. As will subsequently become apparent, this circumstance can be exploited to reduce the domain of analysis to the anharmonic region.

Here we develop a method for effecting this reduction which offers some distinct advantages. We begin by expressing all displacements of the lattice, as the result of unknown forces applied to a perfect harmonic lattice. Because the equilibrating forces are applied to a perfect harmonic lattice, the corresponding displacements can be computed with the aid of the Green function formulated in section 3. Secondly, we partition the lattice into two regions: one containing the defects where anharmonic effects are taken into account, or anharmonic region; and the remainder of the crystal, or harmonic region, where the behavior of the lattice is idealized as being harmonic. It then follows that entire lattice can be equilibrated by applying forces within the anharmonic region only. To establish this fact, let the full lattice contain $N$ atoms (possibly $N=\infty$ ) and let the anharmonic region contain $M<N$ atoms. The equilibrium of the anharmonic region requires

$$
F_{i}^{l}=-\partial \Phi / \partial r_{i}^{l}=0, \quad l=1, \ldots, M
$$

Ciearly, the resulting $2 M$ (non-linear) equations can be satisfied by introducing $2 M$ degrees of freedom in the form of forces applied to the atoms in the anharmonic region. The remaining $2(N-M)$ equations expressing the equilibrium of the atoms in the harmonic region are automatically satisfied by using the Green function for the perfect harmonic lattice to compute displacements. 
The harmonic/anharmonic energy partition construct just described gives the energy of the lattice as

$$
\Phi(1, \ldots, N) \cong \Phi(1, \ldots, M)+\Phi_{\mathrm{L}}^{\mathrm{ext}}
$$

where, for simplicity, we assume than the anharmonic region contains atoms $1, \ldots, M$. An alternative expression for the energy is

$$
\Phi(1, \ldots, N) \cong \Phi(1, \ldots, M)-\Phi_{\mathrm{L}}(1, \ldots, M)+\Phi_{\mathrm{L}}(1, \ldots, N)
$$

where $\Phi_{\mathrm{L}}(1, \ldots, M)$ and $\Phi_{\mathrm{L}}(1, \ldots, N)$ are the harmonic energies of the atoms in the unharmonic region and in the total lattice. Rendering (41) stationary as in (39), the equilibrium equations are found to be of the form

$$
F_{i}^{l}= \begin{cases}\tilde{F}_{i}^{l}-F_{\mathrm{L}_{i}}^{l}+\mathcal{F}_{i}^{M^{\prime}}+\sum_{l^{\prime}=1}^{N} \Phi_{i j}^{N^{n^{\prime}}} u_{j}^{l^{\prime}}-\mathcal{F}_{i}^{N l} & l \leqslant M \\ \sum_{l^{\prime}=1}^{N} \Phi_{i j}^{N^{l^{\prime}}} u_{j}^{l^{\prime}}-\mathcal{F}_{i}^{N^{l}} & l>M\end{cases}
$$

where one writes

$$
\begin{aligned}
& \tilde{F}_{i}^{l}=\frac{\partial \Phi(1, \ldots, M)}{\partial r_{i}^{l}} \\
& F_{\mathrm{L}_{1}}^{l}=\sum_{l^{\prime}=1}^{M} \Phi_{\mathrm{L} i j}^{M^{n^{\prime}}} u_{j}^{l^{\prime}} \quad \Phi_{\mathrm{L} i j}^{M^{\prime^{\prime}}}=\frac{\partial \Phi_{\mathrm{L}}(1, \ldots, M)}{\partial u_{i}^{l}} \\
& \mathcal{F}_{i}^{M^{\prime}}=\sum_{l^{\prime}=1}^{M} \Phi_{L i j}^{M^{l^{\prime}}} \bar{\beta}_{k j}^{l^{\prime}} r_{k}^{l^{\prime}} \\
& \mathcal{F}_{i}^{N^{\prime}}=\sum_{l^{\prime}=1}^{N} \Phi_{L j}^{N^{u l^{\prime}}} \bar{\beta}_{k j}^{l^{\prime}} r_{k}^{l^{\prime}} \quad \Phi_{\mathrm{Lij}}^{N^{l^{\prime}}}=\frac{\partial \Phi_{r m L}(1, \ldots, N)}{\partial u_{i}^{l}} .
\end{aligned}
$$

The system of equations (42) determines the positions $r^{l}$ of the atoms in the relaxed configuration. The displacement field is defined as $u^{l}=r^{l}-r_{0}^{l}$, where $r_{0}^{l}$ represents the initial positions of the atoms on the perfect lattice. It should be carefully noted that, due to the presence of eigendistorsions, the displacements are never uniformly small.

The $2 N$ equations (42) are written in terms of $2 N$ displacement degrees of freedom. To reduce the number of unknowns, we resort to the device of expressing the displacement field as the displacement of a perfect harmonic lattice under the action of fictitious forces $\phi_{j}^{\prime \prime}$ applied to atoms $(1, \ldots, M)$. This gives the representation

$$
u_{i}^{l}=u_{0 t}^{l}+\sum_{l^{\prime}=1}^{M} G_{i j}^{l^{\prime}} \phi_{j}^{l^{\prime}}
$$

where $u_{0 i}^{l}$ represents an initial displacement field and $G_{i j}^{l^{\prime}}$ is the Green function of the perfect harmonic lattice. The initial displacements $u_{0 i}^{l}$ represent macroscopic lattice distorsions such 
as caused by long-range stresses. Finally, in order to obtain a system of equations in $\phi_{j}^{l^{\prime}}$ which derive from a (complementary) potential, we transform (42) into the equivalent set

$$
\begin{aligned}
v_{i}^{l} & \equiv \sum_{l^{\prime}=1}^{N} G_{i j}^{l^{\prime}} F_{j}^{l^{\prime}} \\
& =u_{0 i}^{l}-v_{0 i}^{l}+\sum_{l^{\prime}=1}^{M} G_{i j}^{l^{\prime}}\left(\tilde{F}_{j}^{l^{\prime}}-F_{L_{j}}^{l^{\prime}}+\phi_{j}^{l^{\prime}}\right)=0
\end{aligned}
$$

where

$$
v_{0 i}^{l}=\sum_{l^{\prime}=1}^{M} G_{l j}^{l^{\prime}}\left(\mathcal{F}^{N_{j}^{y^{\prime}}}-\mathcal{F}_{j}^{M^{l^{\prime}}}\right)+\sum_{l^{\prime}=M+1}^{N} G_{i j}^{l^{\prime}} \mathcal{F}_{j}^{N^{l^{\prime}}}
$$

From their definition, it follows that the 'residual displacements' $v_{i}^{l}$ in (45) are simply the displacements which would be obtained by applying the residual forces $F_{j}^{\prime \prime}$ to a perfect harmonic lattice. Clearly, the equilibrium of the lattice requires that $v_{i}^{l}=0$, as stated in (45). Equation (45) is to be solved for the unknowns $\phi_{j}^{\prime \prime}$, which appear explicitly in the last term of (45), and implicitly through the residual $\tilde{F}_{j}^{l^{\prime}}$. Because the displacements $v_{i}^{l}$ derive from a complementary potential, the hessian matrix associated with system (45) is symmetric.

The significance of equation (44) bears some emphasis. Equation (44) merely represents a change of variables from the displacements $u_{i}^{l}$ to the fictitious forces $\phi_{j}^{\prime \prime}$, which are to be determined by minimization of the full potential. The convenience of choosing the fictitious forces $\phi_{j}^{t^{\prime}}$ as primary unknowns stems mainly from the fact that, as already noted, the displacements obtained from (44) are automatically in equilibrium in the harmonic region for all $\phi_{j}^{\prime}$, provided that the initial displacements $u_{0 i}^{l}$ are themselves in equilibrium in that region. In addition, in setting up equations (45), no distinction needs to be made between atoms in the interior and on the boundary of the anharmonic region, which renders the method 'seamless'. Indeed, the equations automatically account for the energy of the harmonic bonds connecting atoms in the harmonic and anharmonic regions.

The obvious parallel between the above procedure and the 'method of forces' of structural mechanics is noteworthy. As in that method, the sought forces $\phi_{j}^{l}$ are those which maximize the complementary energy

$$
\Psi=\sum_{l=1}^{N} \phi_{j}^{l} u_{j}^{l}-\Phi
$$

The introduction of an anharmonic region may then be regarded as a constrained maximization of $\Psi$ subject to the constraints $\phi_{j}^{l}=0, l=M+1, \ldots, N$. This procedure yields a lower bound to the lattice energy, in contrast to displacement methods which bound the energy from above.

The system (45) can be solved by a number of numerical techniques (see, e.g., Dennis and Schnabel 1983, for a review). We have found the Matthies and Strang (1979) factorized form of the BFGS method to be particularly effective. This is a quasi-Newtonian method in which an approximated hessian is updated so as to satisfy a secant condition. Matthies and Strang (1979) expressed the update in a particularly efficient factorized form. QuasiNewtonian methods have the attractive feature of resulting in a quadratic asymptotic rate of 
convergence (Dennis and Schnabel 1983). The number of iterations to convergence can be drastically reduced by preconditioning the hessian by a suitably chosen approximate inverse hessian. In the case of system (45), a readily available and greatly effective approximate inverse hessian is provided by the stiffness matrix $\Phi_{i j}^{I I^{\prime}}$.

\section{Numerical Examples}

As an example of application of the methodology developed in the preceding sections, we consider the problem of a Lomer edge dislocation in silicon (figure 5). While Lomers are not the most common dislocations in silicon, they nevertheless are of some interest for their role in the nucleation of stacking faults, the accommodation of interfacial misfit, the formation of subgrain boundaries and other phenomena (Alexander 1986, Bourret et al 1982, Tan 1981, Honstra 1958). Our focus here, however, is on assessing algorithmic properties of the numerical method such the rate of convergence of the solution with the size of the domain of analysis.

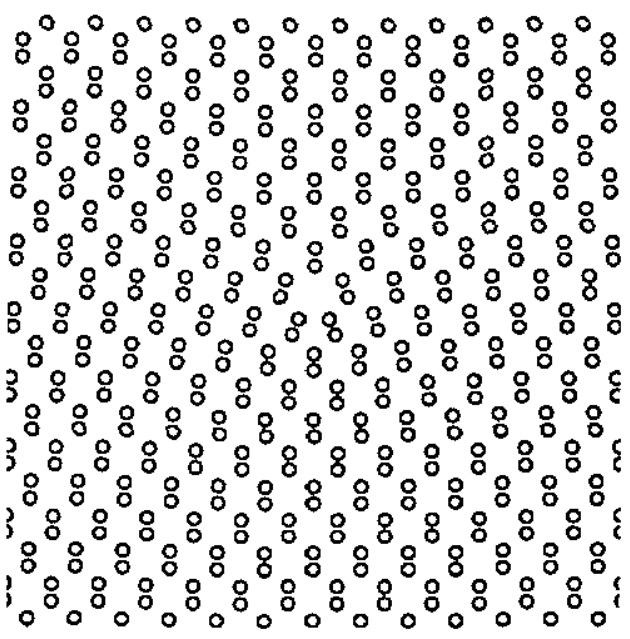

Figure 9. Lomer dislocation in silicon.

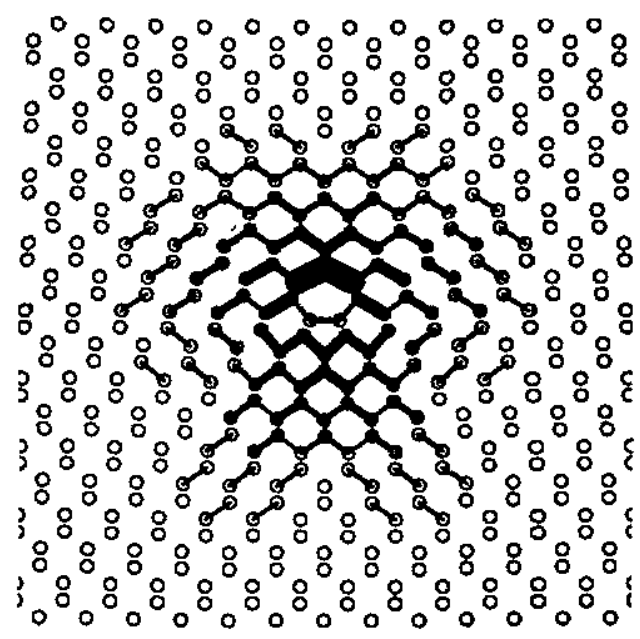

Figure 10. Bond stretching distribution (thickness coded).

The anharmonic structure of the Lomer dislocation computed from Stillinger and Weber's potential is shown in figure 9. The core consists of a pentaring and a heptaring (five-atom ring and seven-atom ring, respectively). Indeed, this is one of the core structures suggested by Hornstra (1958) for edge dislocations on the $\{100\}$ plane, and is characterized by not containing any dangling bonds. Though Lomer dislocations in silicon often dissociate into Shockley partials, they have also been observed undissociated (Bourret et al 1982), which suggests that perfect Lomer dislocations are also stable. It is probable that LomerCottrell locks have a lower energy than perfect Lomer dislocations, which would account for their abundance. It would be of interest to know the magnitude of the energy barrier separating both configurations. These issues lie beyond the numerical scope of this paper and will not be pursued here. 
The solution for the Lomer dislocation bears out the assumption that anharmonic effects are a negligibly small distance away from the core of defects. Figure 10 depicts the bonds which are stretched in excess of $1 \%$ of their equilibrium lengths. The thickness of the bonds in the figure is proportional to their stretching. By this criterion, one concludes that anharmonic effects are confined to a region extending six atomic spacings from the core. The equilibrating forces which in our method account for anharmonic effects are for the most part confined to the same region, with the exception of a 'tail' which extends slightly farther along the slip plane.

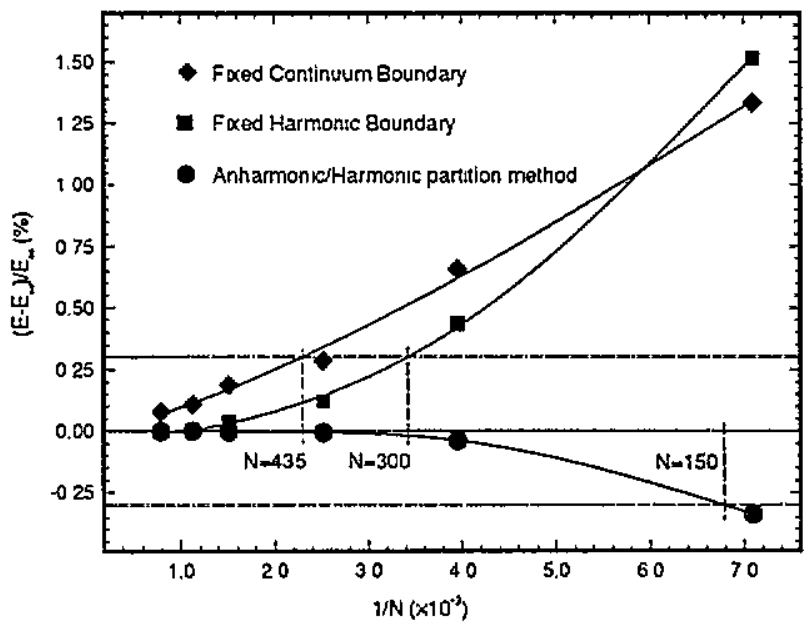

Figure 11. Core energy versus total number of atoms.

Figure 11 compares the convergence properties of the present approach and two fixed boundary methods consisting of prescribing the displacements computed from the harmonic and anisotropic linear elasticity solutions, respectively, on the boundary of the domain of analysis. The harmonic lattice solution for a Lomer dislocation is given by equation (38). For consistency with the rest of the calculations, the anisotropic linear elasticity solution is computed from the elasticities predicted by Stillinger and Weber's potential, equation (10). Errors are measured as the difference between the 'exact' and computed energies of a region of 61 atoms surrounding the core. In lieu of the energy of the exact solution, which is not known, we have obtained an approximate value by extrapolation to $N \rightarrow \infty$. Figure 11 shows the energy error from the three methods as a function of the number of atoms $N$ in the domain of analysis. As expected, the fixed boundary methods converge in energy from above, while the present approach converges from below. The superior accuracy of the present method is evident from the figure.

The rates of convergence of the three methods can be clearly appreciated in the log-log convergence diagram of figure 12. From the slopes of the curves in this diagram it is concluded that the energy error is of $\mathrm{O}\left(N^{-1}\right)$ for the continuum fixed boundary method, of $\mathrm{O}\left(\mathrm{N}^{-2}\right)$ for the hamonic fixed boundary method, and of $\mathrm{O}\left(\mathrm{N}^{-3}\right)$ for the present approach. Thus the rate of convergence of the continuum fixed boundary method is approximately linear, that of the harmonic fixed boundary method is quadratic, and that of the present approach is cubic. As an illustration of the computational implications of these rates of convergence, the number of atoms required by the three methods to obtain solutions within 


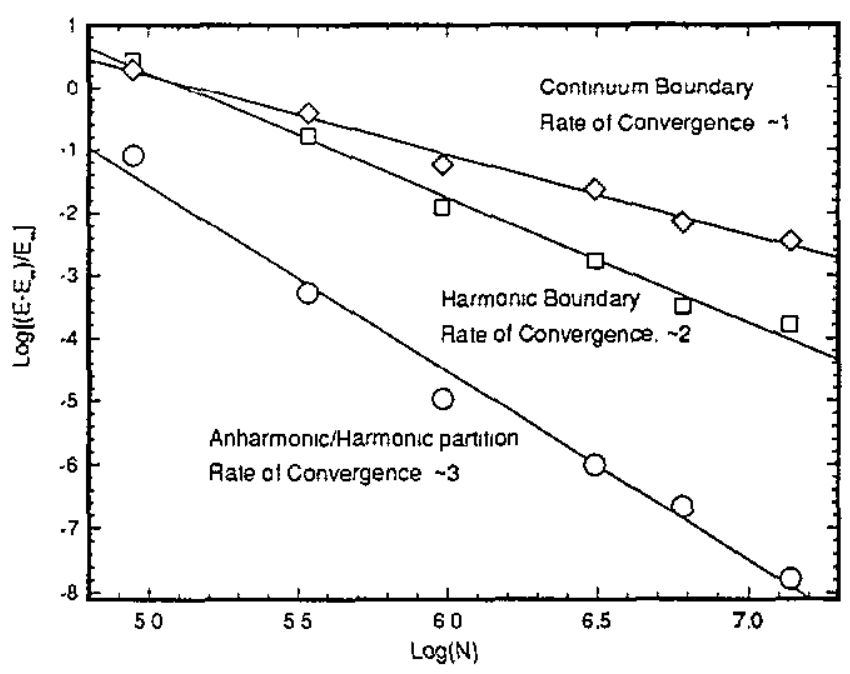

Figure 12. Core energy versus total number of atoms.

$0.3 \%$ of the exact energy in the 61 -atom control region can be read off figure 11 . For the continuum fixed boundary method this gives $N=435$, for the harmonic fixed boundary method $N=300$, and for the present approach $N=150$. These trends can only be expected to be accentuated in three dimensions. Since solution times go as $N^{3}$ for large $N$, it may be concluded that the present approach can result in substantially reduced execution times relative to fixed boundary methods.

\section{Acknowledgments}

MO gratefully acknowledges support from the AFOSR through Grant F49620-92-J-0129. RG is grateful to the Fulbright Comission and the Spanish Education and Science Ministry for the fellowship support provided to carry out his research at Brown University.

\section{References}

Abraham F F and Broughton J Q 1986 Pulsed melting of silicon (111) and (100) surfaces simulated by molecular dynamics Phys. Rev. Lett. 56 734-7

Alexander H 1986 Dislocations in covalent crystals Dislocations in Solids vol 7 ed F R N Nabarro (Amsterdam: North-Holland) pp 114-234

Babŭska I, Vitásek E and Kroupa F 1960 Some applications of the discrete Fourier transform to problems of crystal lattice deformation parts I and II Czech. J. Phys. B 10 419-27, 489-504

Born M and Huang K 1954 Dynamical Theory of Crystal Lattices (London: Oxford University Press)

Bourret A, Desseaux $J$ and Renault A 1982 Core structure of the Lomer dislocation in germanium and silicon Phil. Mag. A bf $451-20$

Boyer L L and Hardy J R 1971 Lattice statics applied to screw dislocations in cubic metals Phil. Mag. 24 647-71 Bullough R and Tewary V K 1979 Lattice theory of dislocations Dislocations in Solids vol 2 ed F R N Nabarro (Amsterdam: North-Holland) pp 1-65

Celli V 1961 Screw dislocation in crystals with diamond structure J. Phys. Chem. Solids $19100-4$

Daw M S 1990 The embedded atom method: a review Many-Atom Interactions in Solids (Springer Proc. Phys. 48)

(Berlin: Springer) pp 49-63 
Dennis J E Jr and Schnabel R B 1983 Numerical methods for unconstrained optimization and nonlinear equations (Englewood Cliffs, NJ: Prentice Hall)

Dodson B W 1986 Evaluation of the Stillinger-Weber classical interaction potential for tetragonal semiconductors in nonideal atomic configurations Phys. Rev. B 33 7361-3

Duesberry M S, Michel D J, Kaxiras E and Joos B 1991 Molecular dynamics studies of defects in Si Mat. Res. Sac. Symp. 209 125-30

Flocken J W and Hardy J R 1970 The method of lattice statics Fundamental Aspects of Dislocation Theory Spec. Publ. 317 vol. 1 ed J A Simmons, R de Witt and R Bullough (National Bureau of Standards) pp 219-45

Gehlen P C, Hirth J P, Hoagland R G and Kanninen M F 1972 A new representation of the strain field associated with the cube-edge dislocation in a model of $\alpha$-iron J. Appl. Phys. 43 3921-32

Haansen P 1983 Electronic processes at dislocation cores and crack tips Atomistic of Fracture (NATO Conf. Ser., Ser. VI: Materials Science) (New York: Plenum) pp 707-30

Halicioglu T, Pamuk H O and Erkoc S 1988 Interatomic potentials with multi-body interactions Phys. Status Solidi b $14981-92$

Heinisch H L Jr and Sines G 1976 A comparison of crystal defect models: I. Displacement of atoms about \{111\} screw dislocations in body-centered cubic metals Phil. Mag. 34 945-60

Hölzer A and Siems R 1970 A lattice theory model for Peierls-energy calculation Fundamental Aspects of Dislocation Theory Spec. Publ. $3 J 7$ vol 1 ed J A Simmons, R de Witt and R Bullough (National Bureau of Standards) pp 291-8

Homstra J 1958 Dislocations in the diamond lattice J. Phys. Chem. Solids $5129-41$

Jones D S 1967 The Theory of Generalised Functions (Cambridge: Cambridge University Press)

Kanzaki H 1957 Point defects in face-centred cubic lattice-distortion around defects J. Phys. Chem. Solids 2 24-36

Landman U, Luedtke W D, Barnett R N. Cleveland C L, Ribarsky M V, Amold E, Ramesh S, Baumgart H, Martinez A and Khan B 1986 Faceting at the silicon (100) crystal-melting interface: theory and experiment Phys. Rev. Lett. $56155-8$

Maradudin A A 1958 Screw dislocations and discrete elastic theory J. Phys. Chem. Solids 9 1-20

Matthies H and Strang G 1979 The solution of nonlinear finite element equations Int. J. Num. Meth. Eng. 14 1613-26

Mura T 1977 Eigenstrains in lattice theory Continuum Models of Discrete Systems (Mont Gabriel, Quebec: University of Waterloo Press) pp 503-19

Nandedkar A S and Narayan J 1990 Atomic structure of dislocations in silicon, germanium and diamond Phil. Mag. A $61873-91$

Simmons G and Wang H 1971 Single Crystal Elastic Constants and Calculated Aggregate Properties: A Handbook, 2nd edn (Cambridge: MIT Press)

Sinclair J E 1971 Improved atomistic model of a bcc dislocation core J. Appl. Phys. 42 5321-9

Sinclair J E, Gehlen P C, Hoagland R G and Hirth J P 1978 Flexible boundary conditions and non-linear geometric effects in atomic dislocation modeling J. Appl. Phys. 49 3890-7

Stillinger F H and Weber T A 1985 Computer simulation of local order in condensed phases of silicon Phys. Rev. B 31 5262-71

Stoneham A M, Torres V T B, Masri P M and Schober H R 1988 Interatomic potentiais in semiconductors and their validity for defect calculations Phil. Mag. A 58 93-106

Stroud A H 1971 Approximate Calculation of Multiple Integrals (Englewood Cliffs, NJ: Prentice Hall)

Tan T Y 1981 Atomic modelling of homogeneous nucleation of dislocations from condensation of point defects in silicon Phil. Mag. A 44 101-25

Teodosiu C 1982 Elastic Models of Crystal Defects (Berlin: Springer)

Tewary V K 1973 Green-function method for lattice statics Advances in Physics 22 757-810

Veyssière P 1988 Dislocation core effects in plasticity Rev. Phys. Appl. 23 431-43

Vitek V 1988 Interactomic forces in relation to the theory of dislocations Phil. Mag. A 58 193-212

Vitek V 1992 Structure of dislocation cores in metallic materials and its impact on their plastic behavior Progress in Material Science vol 36 ed P Haansen and T B Massalski pp 1-27

Wolfram S 1991 Mathematica: A System for Doing Mathematics by Computer (Redwood City, CA: AddisonWesley) 